\title{
A Novel Technique of Branchial Fistula Tract Delineation and Excision In Children Allergic To Dyes
}

\author{
Swagatam Banerjee, ${ }^{1}$ Saurav Sarkar, ${ }^{2}$ Sanjoy Ghosh, ${ }^{1}$ Sharmistha Chakravarty, ${ }^{3}$ Biswajit Sikder ${ }^{4}$
}

\section{Introduction}

\section{ABSTRACT}

Branchial arch fistula which results from a second arch anomaly is rarely seen in practice. The patients usually seek medical advice for persistent discharge from an opening in the neck or mucopurulent discharge from the opening of an infected fistulous tract. The treatment of choice for such a fistula is excision.

\section{Materials and Methods}

A total of seven patients were operated for branchial fistula between 2010 and 2012. Among them, three children could not undergo a fistulogram due to allergy to the dye used and hence they were selected for our modified procedure.

\section{Surgical Procedure}

A 3-0 or 4-0 polypropylene (Prolene ${ }^{\mathrm{TM})}$ thread was inserted into the fistulous tract. Both the ends of the tract were secured with the thread and the sinus tract was dissected out under gentle traction.

$\underline{\text { Results }}$

The patients who were allergic to dye could not have preoperative fistulograms and per-operative injection of dye was also avoided. The blue colour of the polypropylene thread inserted in the fistula tract helped in tracing and excision of the sinus tract. No major complications occurred during or after fistulectomy and the post-operative recovery was uneventful. No recurrence was seen in the scheduled follow-ups.

Conclusion

Surgical excision of branchial fistulas in children with allergy to dyes can be challenging. Insertion of a polypropylene thread into the fistula tract makes its subsequent dissection easy with minimal disruption of adjacent structures.

$\underline{\text { Keywords }}$

Branchial region/abnormalities; Cutaneous fistula/surgery; Fistula/diagnosis

$\mathrm{T}$ The branchial apparatus was first described by von Baer but the anomalies related to its development were described by von Ascheron. ${ }^{1}$ The branchial arches and pouches develop from mesodermal condensations on the lateral aspect of embryonic pharynx. The branchial fistulas arise from abnormalities of the embryonic development of the branchial apparatus.

Branchial arch fistula which results from a second arch anomaly is rarely seen in practice. The treatment of choice for such a fistula is excision.

A branchial fistula arising from the second branchial cleft or pouch usually has an external opening near the anterior border of the ipsilateral sternocleidomastoid muscle in the mid or lower neck region. The fistulous tract usually passes deep to the platysma along the carotid sheath, turns medially and passes distal to the bifurcation of the common carotid artery between the internal and external carotids and crosses the glossopharyngeal and hypoglossal nerves. The internal opening is usually in the ipsilateral tonsillar fossa, on the anterior part of the posterior pillar or in the intratonsillar cleft. ${ }^{2}$

The patients usually seek medical advice for persistent discharge from an opening in the neck or mucopurulent discharge from the opening of an infected fistulous tract.

1 - Department of ENT, Medical College, Kolkata

2 - Department of ENT, AIIMS, Bhubaneswar

3 - Department of ENT, BG\&ID Hospital,Kolkata

4 - Department of ENT, NRS Medical College, Kolkata

Corresponding author:

Dr Swagatam Banerjee

email: drswagatam@gmail.com 
In most cases, the pathway described earlier is not completely present, ${ }^{3}$ the common presentation being a tract extending up the neck for a varying distance with a simple sinus opening. The extent of the tract can be delineated with a dye test or fistulogram keeping in mind that an incomplete tract in preoperative testing may turn out to be a complete one peroperatively due to the effects of muscle relaxants during general anaesthesia. ${ }^{2}$

\section{Materials and Methods}

A total of seven patients were operated for branchial fistula between 2010 and 2012. A diagnosis of branchial arch fistula was made in all these cases but the three children in this study could not undergo a fistulogram due to allergy to the dye used and hence they were selected for this procedure.

\section{Surgical Procedure}

Under general anaesthesia with nasotracheal intubation, the neck and mouth were prepared as one operative field. The cervical opening of the fistulous tract was dilated with a Nettleship's punctum dilator. A 3-0 or 4-0 polypropylene (Prolene ${ }^{\mathrm{TM}}$ ) thread was then inserted into the cervical opening and passed via the fistulous tract as shown in Fig. 1. Of the three children who underwent this procedure, two were found to have complete tracts with the polypropylene thread coming out from the other end (intraoral) Fig. 2, while one had an incomplete tract.

In case of the incomplete fistulous tract, after inserting the polypropylene thread as much as possible, the cervical opening was incised around the polypropylene thread and proximal dissection done. The polypropylene thread was fixed in its position in the tract by tying a silk knot around it. The blue colour of the polypropylene thread helped in tracing the sinus tract and thus helped in complete excision of the tract.

In the complete fistulous tracts which were opening intraorally, the polypropylene thread was inserted after prior dilatation of the cervical opening as mentioned above. At the oral site, a small mucosal incision was made around the end of the polypropylene thread. The dissection of the parapharyngeal segment of the fistula, which was usually a few millimeters in length, was

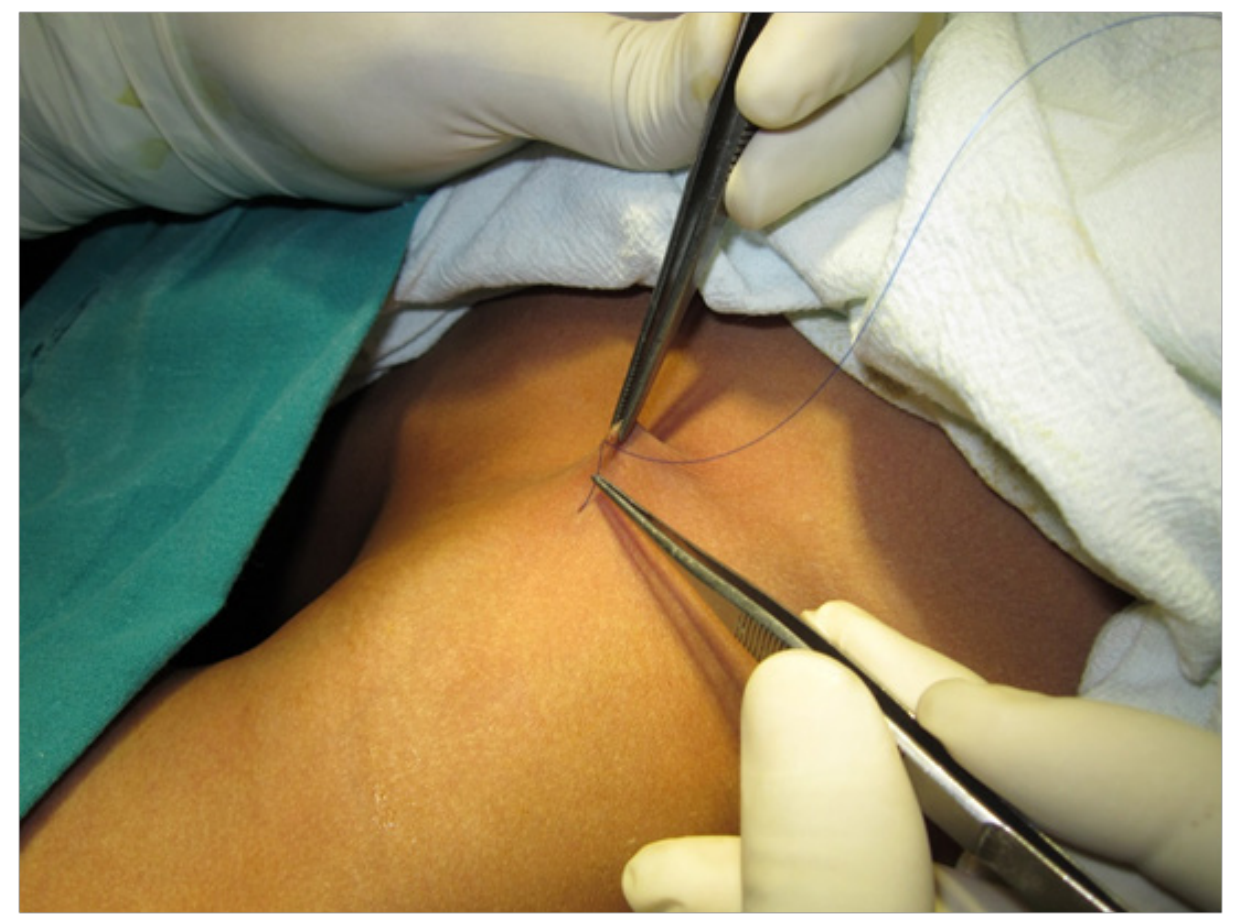

Fig. 1 4-0 Polypropylene suture material being inserted in the cervical opening of the fistulous tract 


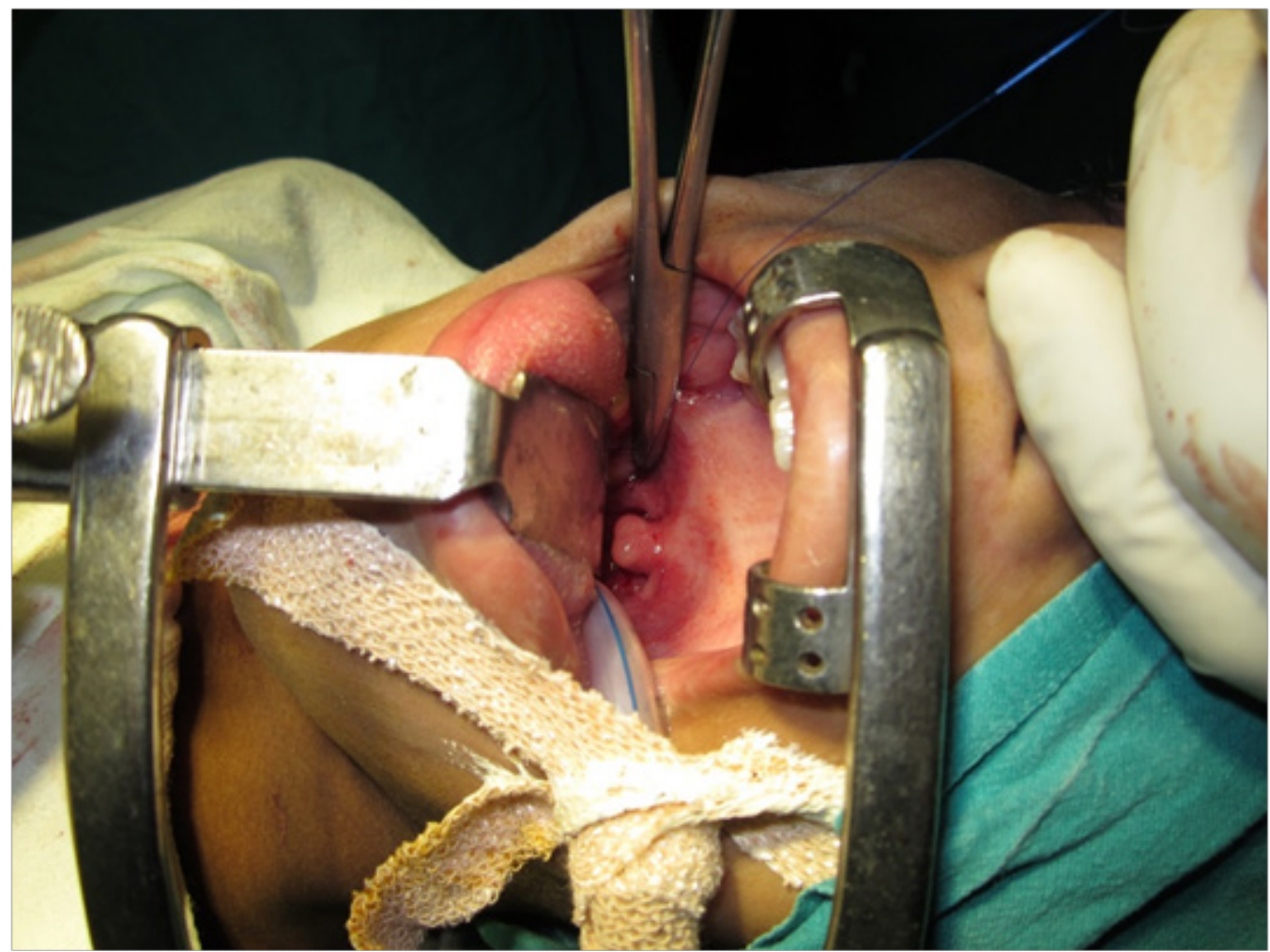

Fig. 2 Polypropylene thread coming out from the other end - intraoral, posterior pillar of tonsil in complete branchial fistula

carried out via the oral site using forceps. The oral part of the fistulous tract was tied over the polypropylene thread with 4-0 silk. Then, it was gently pulled from the neck site resulting in the fistula being straightened and shortened to half its length. The first few millimetres of the cervical portion of the fistula was freed by blunt dissection. Then, with a 3-0 or 4-0 silk thread, it was transfixed to the dissected tract. Under the gentle traction of both polypropylene and silk threads, only the fistula tract was carefully dissected from the surrounding structures, ultimately reaching the already dissected out portion from the oral side. The blue colour of the polypropylene thread served as a good guide for dissection of the tract. Thus, the entire tract was pulled out over the polypropylene thread and delivered entirely through the neck (Fig. 3).

\section{Results}

The patients comprised two boys and a girl, their ages being 4 years, 6 years and 3 years respectively. All the patients were allergic to dye, so preoperative fistulogram was not done and per-operative injection of dye was also avoided. No major complications occurred during or after fistulectomy. Histological examination showed a fistula tract with a muscular wall lined by benign squamous epithelia.

Fistula in the third case was found to be incomplete per-operatively. The post-operative recovery was uneventful. No recurrence was seen in the scheduled follow-ups.

\section{Discussion}

Embryonic developmental anomalies resulting in pharyngeal arch fistulas and cysts are usually uncommon. ${ }^{1}$ However, among these, second arch anomalies are probably the most common and may manifest in various forms including a complete fistulous tract with external and internal openings, albeit rarely. It 


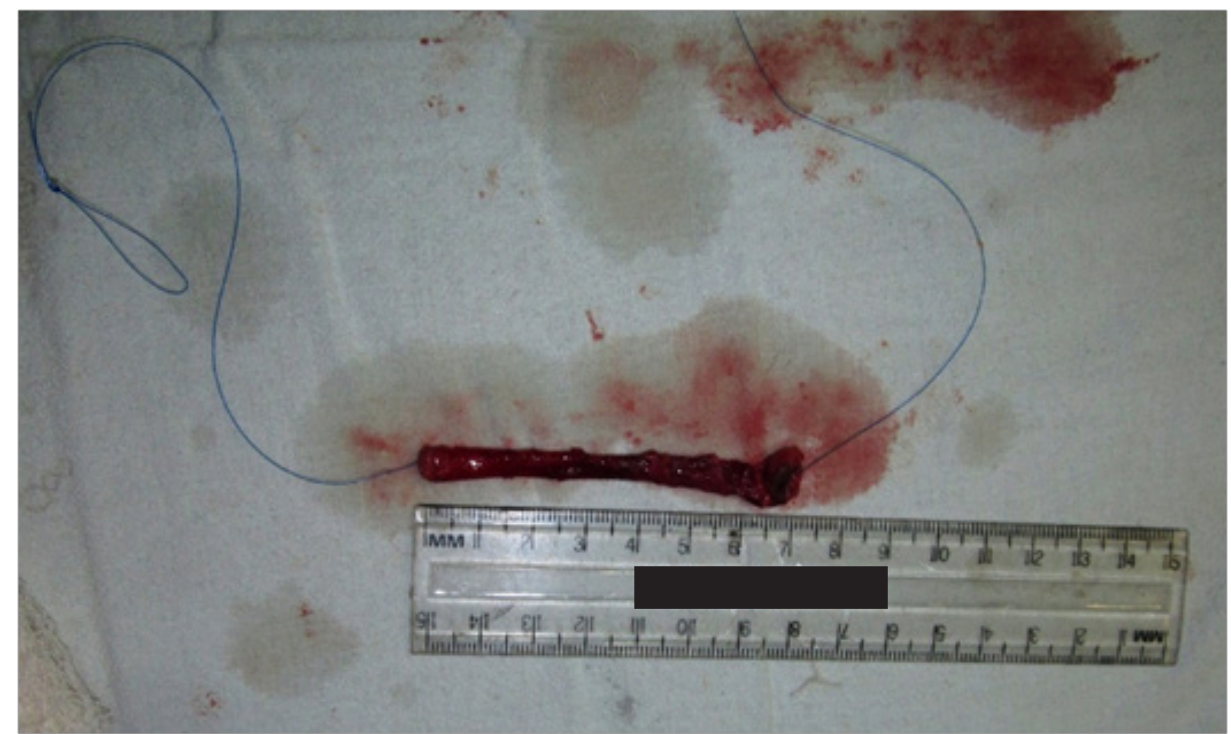

Fig. 3 The entire fistulous tract along with polypropylene thread

usually has an external opening near the anterior border of the ipsilateral sternocleidomastoid muscle in the mid or lower neck region. The fistulous tract passes deep to the platysma along the carotid sheath, turns medially and passes distal to the bifurcation of the common carotid artery between the internal and external carotids and crosses the glossopharyngeal and hypoglossal nerves. The internal opening is usually in the ipsilateral tonsillar fossa, on the anterior part of the posterior pillar or in the intratonsillar cleft.

Earlier, branchial fistulas were treated by injection of sclerosing agents to obliterate the tract with minimal scarring. However, the adjacent structures were quite often at risk because of the severe reaction produced. ${ }^{4}$ Hence, total surgical excision of the fistulous tract is the mainstay of modern treatment. Amongst the various techniques described for surgical excision, the standard one involving stepladder incisions as described by Bailey in 1933 is commonly used. ${ }^{4,5}$ Other methods described in medical literature include a pull-through branchial fistulectomy ${ }^{2}$ and use of arterial intimal strippers. ${ }^{4,6,7}$ However, these methods are not in common practice despite having cosmetic benefits over the conventional stepladder procedure. A procedure involving the insertion of a nylon thread into the fistulous tract was described by Azuma et al in $1986 .^{8}$ In our study, polypropelene was used as it is coloured which helped in better delineation of the tract peroperative.

\section{Conclusion}

Surgical excision of branchial fistulas in children with allergy to dyes can be challenging because of the difficulty in delineation of the fistulous tract. Insertion of a 3-0 or 4-0 polypropylene (Prolene ${ }^{\mathrm{TM}}$ ) thread into the fistula tract makes its subsequent dissection easy with minimal disruption of adjacent structures. Thus, it is a good alternative of fistulous tract delineation in children specially avoiding dyes and radiation during fistulogram.

\section{References:}

1. De PR, Mikhail T. A combined approach excision of branchial fistula. J Laryngol Otol 1995;109:999-1000

2. Talaat M. Pull-through branchial fistulectomy: technique for the otolaryngologist. Ann Otol Rhino Laryngol 1992;101:501-2

3. Ford GR, Balakrishnan A, Evans JN, et al. Branchial cleft and pouch anomalies. J Laryngol Otol 1992;106:137-43

4. Bailey H. The clinical aspects of branchial fistula. Br J Surg 1933;21:173- 82

5. Taylor PH, Bicknell PG. Stripping of branchial fistulae, a new technique. J Laryngol Otol 1977;91:141- 9 
6. Cox EF. Excision of branchial sinus and fistula tracts using arterial intimal strippers. Surg Gynecol Obstet 1963;117:767-8

7. Lee STS, Krishnan MMS. Branchial fistula- a review. Singapore Med J 1991;32:50-2
8. Azuma T, Nagahara S, Kawata H, et al. Surgery for complete type branchial fistula with insertion of a nylon thread. J Jap Soc Pediatr Surgeon 1986;327 